\title{
A Rare Localisation of Osteoid Osteoma in a Young Male
}

\author{
Louise Hvid Kristiansen $^{1 *}$, Jens Tvedegaard-Christensen ${ }^{1}$ and Rikke Thorninger ${ }^{1,2}$ \\ ${ }^{1}$ Department of orthopaedic surgery, Regional Hospital Randers, Central Region of Denmark, Skovlyvej 15, 8930 Randers NØ, Denmark \\ 2Institute of Clinical Medicine, Aarhus University, Palle Juul-Jens Boulevard 99, 8200 Aarhus N, Denmark.
}

${ }^{\star}$ Corresponding author: Louise Hvid Kristiansen, Department of orthopaedic surgery, Regional Hospital Randers, Central Region of Denmark, Skovlyvej 15, 8930 Randers NØ, Aeblerosevej 5, 8541 Skoedstrup, Denmark; Phone: +4528593727; Email: annlkris@rm.dk

Received: October 14, 2019; Accepted: October 23, 2019; Published: October 29, 2019;

\begin{abstract}
Osteoid Osteoma is a benign bone tumor accounting for 10-12\% of all benign bone tumors. There is an evident male: female ratio of $2: 1$. The far most common site of location is the long bones of the lower extremities, which accounts for approximately $50 \%$ of all osteoid osteomas. Osteoid osteoma in the hand is a rare finding, accounting for only six to $13 \%$. When found in the hand, the phalanges are the most frequent localisation followed by the carpals. The metacarpals are the least frequent site of location of $\mathrm{OO}$ in the hand.

We present a case of a 19-year-old male with characteristic symptoms of osteoid osteoma during a nine-month period. The patient presented with longterm pain at the tumor site with nocturnal worsening. Plain radiography and MR showed a characteristic nidus suggesting the diagnosis of osteoid osteoma.

Treatment of osteoid osteoma can be non-surgical treatment with the use of non-steroidal anti-inflammatory drugs. Since few reports have suggested a possible transformation of osteoid osteoma into malignant osteoblastoma, most patients are treated surgically with en-bloc resection either open or CT-guided depending on local resources.
\end{abstract}

Keywords: Osteoid osteoma; Metacarpal Bone; Rare Localisation

\section{Introduction}

Osteoid Osteoma (OO) accounts for approximately 10-12\% of all benign bone tumors [1]. The tumors are most often localised in the long bone of the lower extremities. Localisation of the hand accounts for only $10 \%$. Of these the phalanges are the most frequent localisation followed by the carpals. The metacarpals are the least frequent site of localisation in the hand.

We present a case of a very rare localisation of osteoid osteoma in the metacarpal bone of a young male.

\section{Case}

We present a case of osteoid osteoma localised in the first metacarpal bone of a 19-year old male initially examined in our ambulatory care unit in august 2019 .

The patient presented with symptoms of long-term localised pain at the first metacarpal bone of the right hand. The patient reported increasing pain at night with intermittent awakenings due to pain. Pain had evolved during the past nine months. The patient noticed no swelling, tenderness or restricted range of motion. At the clinical examination neither swelling nor decreased range of motion was observed. There were no sensory disturbances and no reduction of strength or function. No history of trauma or family history of OO was present either.
Initially a plain radiography was obtained showing a characteristic nidus with periostal thickening raising a high suspicion of osteoid osteoma despite its rare localisation (see figure 1-2).

An additional MR scan with contrast was obtained afterwards also suggesting the suspected diagnosis of osteoid osteoma. Final diagnosis is only possible to obtain after histological examination postoperatively.

In this case the nidus of the OO was obvious on the initial radiography and we decided to perform only an MR to exclude other malignant differential diagnosis (see figure 3-4)

The patient was discussed in a multicenter sarcoma conference and it was decided to offer the patient surgical removal of the ostoid osteoma by curretage in another hospital.

\section{Discussion}

Osteoid osteoma is a benign bone-forming tumor first described in 1935 by Jaffe [2]. Osteoid osteoma accounts for approximately 10-12\% of all benign bone tumors [1]. There is an evident male predilection with a male: female ratio of $2: 1$. OO is most often diagnosed in the second decade of life, principally between 7 and 25 years of age, peaking around 15 years of age [3-5].

The far most common site of location for OO is the long bones of the lower extremities, particularly femur and tibia. These localisations account for approximately $50 \%$ of all osteoid osteomas [1]. 10\% are localised in the spinal column [3]. 


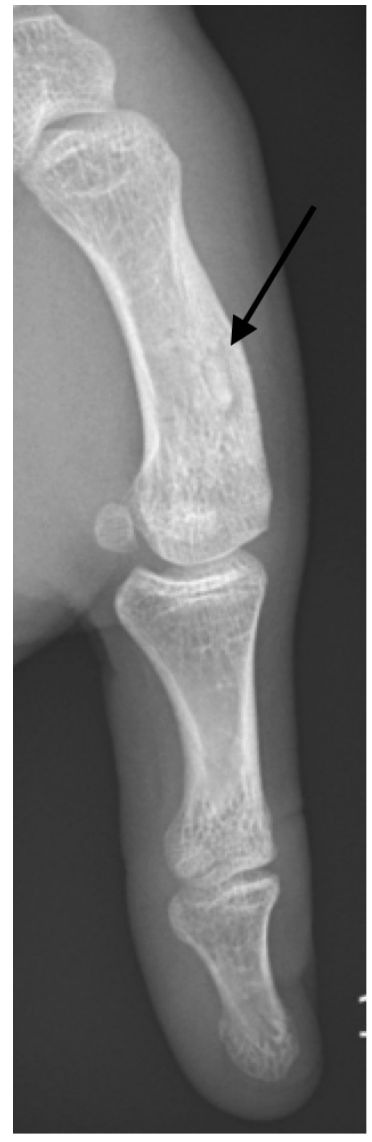

Figure 1

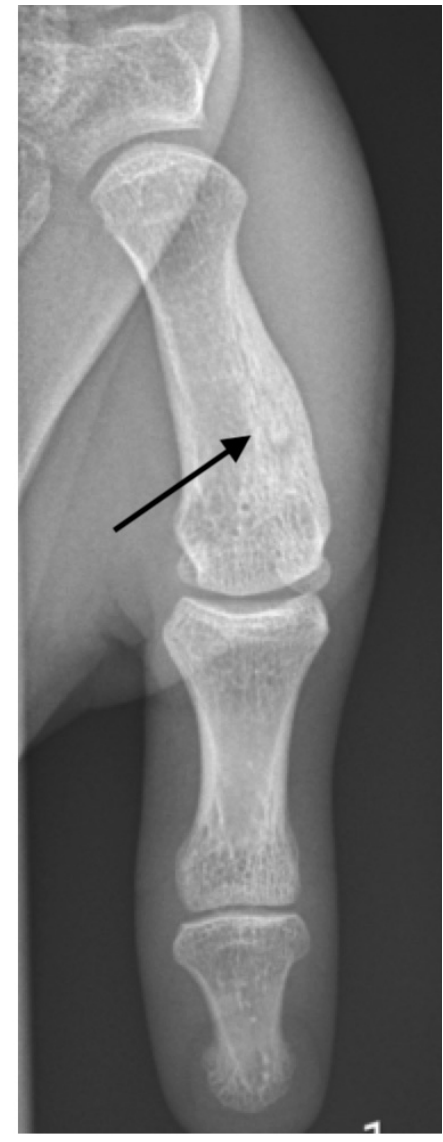

Figure 2
Figure 1 and 2. Radiographies obtained initially showing an arrow pointing at the characteristic nidus.

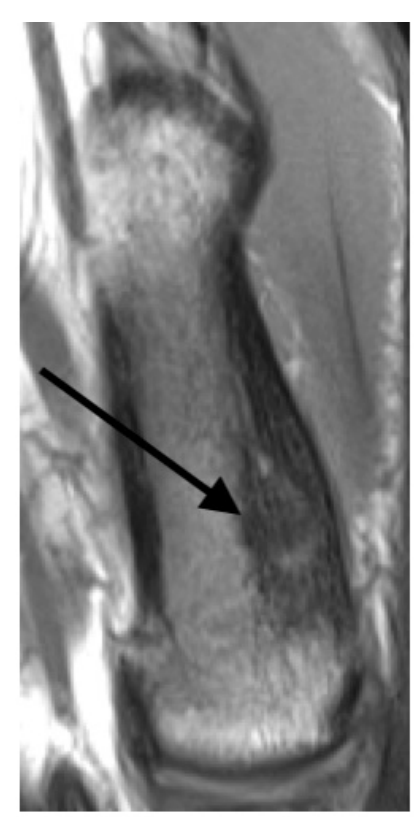

Figure 3

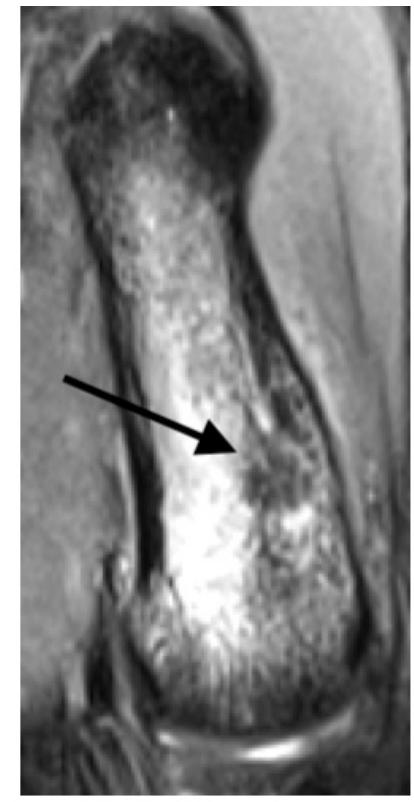

Figure 4
Figure 3 and 4. MR T1 sequences with intravenous contrast showing a characteristic nidus, approximately $4 \mathrm{~mm}$ in diameter. Thickening of the cortex in the distal $2 / 3$ of the metacarpal bone.

Evaluation by a radiologist also suggests osteoid osteoma.
Localisation of $\mathrm{OO}$ in the hand is a rare finding accounting for only six to $13 \%$ of all osteoid osteomas [5]. When found in the hand, the phalanges are the most frequent localisation followed by the carpals. The metacarpals are the least frequent site of location of $\mathrm{OO}$ in the hand [1]. The tumor is most frequently localised in the cortical bone in the diaphysis or metaphysis [1].

The etiology for $\mathrm{OO}$ is unknown but some studies suggest familiar disposition and trauma as possible causes [6,8]. Exact pathogenesis of $\mathrm{OO}$ also remains unknown. Vasodilation and local inflammation at the tumor site is thought to be caused by prostaglandin E2 and prostacyclin found within the nidus [3,9] Former studies demonstrated bundles of nerve fibres within the nidus contributing to pain and local oedema stimulated by prostaglandins within the nidus [1].

First suspicion of OO most often occurs due to the characteristic symptoms including an initial plain radiography.

In plain radiography $\mathrm{OO}$ appears as an oval lytic lesions surrounded by bone thickening and sclerosis. However the central nidus is not always apparent [3]. Not all OO lesions can be diagnosed in radiography, but this is often the primary modality of choice. Further imaging modalities are necessary if there is a high suspicion of $\mathrm{OO}$, although there is no finding on plain radiography [3].

Computed Tomography (CT) is considered the modality of choice in diagnosing $\mathrm{OO}$ to distinguish the lesion from other differential diagnosis. The nidus is visualised as a low-attenuated central zone with variable surrounding sclerosis. CT is an important imaging method when plain radiography does not reveal the lesion or with intraarticular lesions.

Visualisation of the OO lesion is very variable with MR diagnostics [1]. Compared to MR, CT is more specific in identifying a nidus [3]. MR benefits from its ability to detect soft tissue involvement. If the nidus is located close to the medullary zone MR has a greater role in diagnosing and visualising the lesion compared to CT. When using $\mathrm{MR}$ as the only imaging modality there is a potential of misdiagnosis or overseeing the lesion [3].

Other imaging modalities that can be used in diagnosing $\mathrm{OO}$ is bone scintigraphy and PET scans.

In this case the nidus of the OO was very obvious on the initial radiography and we decided to perform only an MR to exclude other malignant differential diagnosis.

Since the etiology of $\mathrm{OO}$ is non-malignant and the lesion has a history of spontaneous healing non-surgical treatment can be considered. Non-Steroidal Anti-Inflammatory Drugs (NSAID's) are proven effective in relieving pain, according to the presence of prostaglandin production in the nidus. Side effects of NSAID's are an important consideration in long time treatment. Knowledge according to prolonged medical use in treating OO lesions lacks. Studies recommend caution using NSAID's due to lack of knowledge of long-time use [1,3].

Few reports have suggested that $\mathrm{OO}$ can progress to malignant osteoblastoma with prolonged NSAID use [10]. Non-surgical management is a justifiable treatment if the localisation of the lesions 
is for example intraarticular or difficult to remove or if the patient is not willing to go through surgery.

Surgical procedures are suitable for patients not responding to medical treatment, patients with severe pain or patients not willing to accept possible long-term effects of NSAID treatment. Children with remaining growth potential and open physes in risk of limb-length discrepancy scoliosis and osteoarthritis are also surgical candidates [3].

En bloc resection with complete resection of the total nidus is a frequently used surgical option. For complete pain relive and minimal risk of recurrence it is necessary to resect the entire nidus. Resection of the surrounding sclerotic bone is not required [3]. The use of this procedure may leave behind a bone defect possibly requiring bone grafting or internal fixation. Postoperative restrictions in activity and weight bearing are often recommended after en bloc resection [3]. Challenges in using this technique are difficulties of identifying the lesion perioperatively, possibly resulting in incomplete resection or removal of too much bone tissue [1].

CT guided percutaneous excision is an increasingly used alternative surgical procedure due to reduced morbidity. The procedures include cryoablation, radiofrequency ablation and laser thermocoagulation amongst others. The possibility of perioperative CT visualisation is a great advantage compared to the open en bloc resection. Several studies have shown good results with minimal relapses, low morbidity and few postoperative restrictions. The procedure is though not available in all hospitals $[1,3]$.

Irrespective of what technique is used biopsies are always required to confirm the diagnosis.

This particular patient was offered open curretage of the lesion in another hospital with a sarcoma center. The patient has not yet decided whether to accept the offer of surgery.

Only a few other cases have presented OO localised in the metacarpal bone, which makes this case extraordinary. When patients present with the characteristic symptoms of localised pain and nocturnal worsening in the young male population it is important to remember that $\mathrm{OO}$ can present in upper extremity though very rare. Plain radiography is always a simple initial examination to perform.

The patient involved in this case report gave consent to the use of the patient history in this article.

\section{References}

1. Atesok KI, Alman BA, Schemitsch EH, Peyser A et al. (2011) Osteoid osteoma and osteoblastoma. J Am Acad Orthop Surg 19: 678-689.

2. JAFFE HL. Osteoid-osteoma. Proc R Soc Med 46: 1007-1012.

3. Noordin S, Allana S, Hilal K, et al. (2018) Osteoid osteoma: Contemporary management. Orthop Rev (Pavia) 10: 7496.

4. El Fatayri B, Djebara AE, Fourdrain A, Bulaid Y et al (2019) Resection of a rare metacarpal distal condyle osteoid osteoma. Case Rep Orthop 2019: 4542862.

5. Brohard J, Tsai P (2019) Osteoid osteoma in the thumb of an adolescent patient. $J$ Hand Surg Am 2019.

6. Seker A, Unal MB, Malkoc M, Kara A et al. (2016) A rare localization of osteoid osteoma - presentation of two cases. Srp Arh Celok Lek 144: 553-556.

7. Chronopoulos E, Xypnitos FN, Nikolaou VS, Efstathopoulos N et al. (2008) Osteoid osteoma of a metacarpal bone: A case report and review of the literature. $J$ Med Case Rep 2: 285-1947-2-285.
8. Kalil RK, Antunes JS (2003) Familial occurrence of osteoid osteoma. Skeletal Radiol 32: 416-419.

9. Makley JT, Dunn MJ (1982) Prostaglandin synthesis by osteoid osteoma. Lancet 2: 42-6736(82)91174-6.

10. Bruneau M, Polivka M, Cornelius JF, George B (2005) Progression of an osteoid osteoma to an osteoblastoma. case report. J Neurosurg Spine 3: 238-241.
Citation:

Kristiansen LH, Tvedegaard-Christensen J, Thorninger R (2019) A Rare Localisation of Osteoid Osteoma in a Young Male. Integr J Orthop Traumatol Volume 2 (5): 1-3. 